\title{
Forkhead box P3 promotes breast cancer cell apoptosis by regulating programmed cell death 4 expression
}

\author{
DONG FAN ${ }^{1,2^{*}}$, CHENG ZENG $^{3,4^{*}}$, SHUMING WANG $^{5 *}$, JUN HAN $^{1}$, LIAOLIAO ZHU $^{1}$, \\ HUADONG ZHAO ${ }^{2}$, YINGQI ZHANG ${ }^{1}$, JIANGUO LU ${ }^{2}$ and YING XU ${ }^{1,6}$
}

\begin{abstract}
${ }^{1}$ State Key Laboratory of Cancer Biology, Biotechnology Center, School of Pharmacy, Air Force Medical University, Xi'an, Shaanxi 710032; ${ }^{2}$ Department of General Surgery, Tangdu Hospital, Air Force Medical University, Xi'an, Shaanxi 710038;

${ }^{3}$ Department of Chinese Materia Medical and Natural Medicines, School of Pharmacy, Air Force Medical University, Xi'an, Shaanxi 710032; ${ }^{4}$ Department of Pharmacy, Central Theater Command Air Force Hospital, Datong, Shaanxi 037000; ${ }^{5}$ Outpatient Department of Rocket Engineering University, The 986th Hospital of Air Force, Air Force Medical University, Xi'an, Shaanxi 710054; ${ }^{6}$ Department of Obstetrics and Gynecology, The First Affiliated Hospital of The Air Force Medical University, Xi'an, Shaanxi 710032, P.R. China
\end{abstract}

Received November 19, 2019; Accepted August 18, 2020

DOI: 10.3892/ol.2020.12155

\begin{abstract}
Forkhead box P3 (FOXP3), an X-linked tumor suppressor gene, plays an important role in breast cancer. However, the biological functions of FOXP3 in breast cancer apoptosis remain unclear. To investigate the underlying genes and networks regulated by FOXP3 in breast cancer, RNA sequencing was performed to compare FOXP3-overexpressing MDA-MB-231 cells and control MDA-MB-231 cells. Differentially expressed genes were identified, and functional enrichment analysis comparing the two groups was performed. The differentially expressed genes were mainly enriched in phagosomes, oxytocin, serotonergic synapses and the phospholipase D signaling pathway. Furthermore, gene set enrichment analysis revealed the enrichment of a gene signature associated with apoptosis in FOXP3-overexpressing MDA-MB-231 cells compared with wild-type cells. Further analysis showed that programmed cell death 4 (PDCD4), a key molecule involved in apoptosis, was overexpressed in FOXP3-MDA-MB-231 cells. Reverse transcription-quantitative PCR and western
\end{abstract}

Correspondence to: Mrs. Ying $\mathrm{Xu}$, State Key Laboratory of Cancer Biology, Biotechnology Center, School of Pharmacy, Air Force Medical University, 169 Changle Road (West), Xi'an, Shaanxi 710032, P.R. China

E-mail: xuyinglc@126.com

Dr Jianguo Lu, Department of General Surgery, Tangdu Hospital, Air Force Medical University, 569 Xinsi Road, Xi'an, Shaanxi 710038, P.R. China

E-mail: lujguo@fmmu.edu.cn

${ }^{*}$ Contributed equally

Key words: RNA-seq, breast cancer, forkhead box P3, apoptosis, programmed cell death 4 , differentially expressed genes blotting showed that FOXP3 upregulated the expression of PDCD4 in breast cancer cells. Clinical sample analysis using a public database showed that the expression level of PDCD4 was associated with breast cancer clinical stages. Overall, the present study suggested that FOXP3 can promote the apoptosis of breast cancer cells by upregulating the expression of PDCD4, thus exerting a tumor suppressive function.

\section{Introduction}

Breast cancer is a type of malignant tumor originating from breast epithelial tissue that accounts for one-fourth of cancer cases diagnosed in females in 2018 worldwide and that seriously endangers women's health (1). In recent years, with the increase in early screening and the improvement of treatment, the survival and prognosis of breast cancer have improved, but the overall curative ratio is still not ideal (2-4). Based on statistics, there were $\sim 1.7$ million new cases of breast cancer and 520,000 breast cancer-associated deaths worldwide in 2012 (5), while by 2018, the number of new cases of breast cancer increased to $\sim 2.1$ million, and the number of deaths was $\sim 630,000$ (2). With the changes in lifestyle and reproductive choices, the incidence and mortality rate of breast cancer are increasing, and breast cancer is becoming a serious global health burden (6).

The overactivation of oncogenes and the inactivation of tumor suppressor genes are important causes of the occurrence and development of breast cancer (7). Among these genes, forkhead box P3 (FOXP3) plays an important suppressive role in breast cancer. FOXP3 is a specific marker of regulatory $\mathrm{T}$ cells, and this gene plays an important role in the differentiation, development and functional maintenance of $\mathrm{T}$ regulatory cells (8-11). A large number of studies have shown that FOXP3 is also expressed in normal breast epithelial cells and is an important X-linked breast cancer suppressor gene that plays an important role in the metastatic spread of breast cancer by regulating the expression of a series of tumor-related genes, 
including BRCA1/2, HER2, c-myc and SKP2 (12-14). The deletion, mutation and change of the cellular localization of FOXP3 are important causes of the occurrence and development of breast cancer (12). In our previous study, it was reported that FOXP3 inhibits breast cancer angiogenesis by regulating the expression of VEGF (15). However, it remains unclear whether FOXP3 is involved in the regulation of breast cancer cell apoptosis. The present study aimed to investigate the underlying genes and networks regulated by FOXP3 in breast cancer.

\section{Materials and methods}

Cell lines and culture. The human breast cancer cell lines MCF-7 and MDA-MB-231 were obtained from The Cell Bank of Type Culture Collection of the Chinese Academy of Sciences. All cell lines were authenticated by the analysis of short tandem repeat profiles and $100 \%$ matched the standard cell lines in the DSMZ data bank. All cells were negative for the cross-contamination of other human cells and for mycoplasma contamination. The cells were cultured in DMEM medium (Gibco; Thermo Fisher Scientific, Inc.) with $10 \%$ estrogen-deprived fetal bovine serum (HyClone; Cyvita) and $100 \mathrm{mg}$ per $\mathrm{ml}$ ampicillin/streptomycin.

Generation of FOXP3-MDA-MB-231 cells. FOXP3-overexpresing lentivirus was constructed and purchased from GeneChem, Inc. In total, 3x105 MDA-MB-231 cells were seeded onto a six-well culture plate, and the second-generation lentivirus-containing control vector or a FOXP3 vector (MOI 10) was added to the plate. After $12 \mathrm{~h}$, the medium was replaced, and cells were cultured in incubator of $37^{\circ} \mathrm{C}$ for $96 \mathrm{~h}$. Puromycin $(2 \mu \mathrm{g} / \mathrm{ml})$ was used to select infected cells. MDA-MB-231 breast cancer cells were infected with GFP-labeled FOXP3-overexpressing adenovirus or control adenovirus, and the bright field and eGFP expression patterns (representing cells that were effectively infected with adenovirus) were examined using fluorescence microscopy at x20 magnification.

Plasmid construction and RNA interference. XhoI and KpnI flanked HFOXP3 were synthesized by TsingKe Biological Technology, and digested HFOXP3 was subcloned into identically digested pcDNA3.1+ to generated pcDNA3.1-FOXP3 plasmid. The FOXP3 siRNA (sense 5'-GCAGCGGACACU CAAUGAGdTdT-3'; antisense 5'-CUCUUUGUGUGUCCG CUGCdTdT-3') (16) and the negative control (sense 5'-GCA GCGGACACUCAAUGAGdTdT-3'; antisense 5'-CUCUUU GUGUGUCCGCUGCdTdT-3') were purchased from Shanghai GenePharma Co., Ltd. The pcDNA3.1-FOXP3 plasmid and FOXP3 siRNA were used to overexpress and knockdown FOXP3 expression, respectively, in MDA-MB-231and MCF-7 cells. The PDCD4 siRNA (sense, 5'-GCUGCUUUGGAC AAGGCUATT-3'; antisense, 5'-UAGCCUUGUCCAAAG CAGCTT-3') and the negative control (sense, 5'-GCUGCU UTGGACAAGGCUATC-3'; antisense, 5'-UAGCCUAGU CCAAAGCAGCAT-3') sequences (17) were synthesized by Shanghai GenePharma Co., Ltd.

Cell transfection. Breast cancer cells MDA-MB-231 and MCF-7 were seeded in 6-well plate at the density of $2 \times 10^{5}$ cells/well and cultured overnight at $37^{\circ} \mathrm{C}$. Once cell density reached $70 \%$, the culture medium was discarded and OPTI-MEM (Gibco; Thermo Fisher Scientific, Inc.) was added for another $4 \mathrm{~h}$; the pcDNA3.1-FOXP3 plasmid $(2.5 \mu \mathrm{g})$ and siRNA (MOI 20, $5 \mu \mathrm{l}$ ) were transfected into MDA-MB-231 and MCF-7 cells, respectively, using Lipofectamine 3000 (Invitrogen; Thermo Fisher Scientific, Inc.) following the manufacturer's instructions. After $6 \mathrm{~h}$ incubation at $37^{\circ} \mathrm{C}$, the medium was replaced and the cells were cultured in appropriate DMEM medium (Gibco; Thermo Fisher Scientific, Inc.) supplemented with $10 \%$ estrogen-deprived fetal bovine serum (HyClone; Cyvita) for various time periods. At the same time, cells were transfected with control siRNA as control group.

Reverse transcription-quantitative PCR. Total RNA was isolated from cells with RNAIso Plus (Takara Biotechnology Co., Ltd.), and RNA (100 ng) was reverse transcribed into single stranded cDNA using a PrimeScript RT Reagent kit (Takara Biotechnology Co., Ltd.) in $10 \mu$ l reaction at $37 \mathrm{C}$ for $15 \mathrm{~min}, 85^{\circ} \mathrm{C}$ for $5 \mathrm{~min}, 4^{\circ} \mathrm{C}$ for hold. Then, $2 \mu 1 \mathrm{cDNA}$ was used for qPCR using a Prism 7500 real-time thermocycler (Applied Biosystems; Thermo Fisher Scientific, Inc.) using with SYBR Green Ex Taq (Takara Biotechnology Co., Ltd.) according to the manufacturer's instructions. The reaction protocol was as follows: $30 \mathrm{sec}$ at $95^{\circ} \mathrm{C}$, followed by 40 cycles of $5 \mathrm{sec}$ at $95^{\circ} \mathrm{C}$ and $34 \mathrm{sec}$ at $60^{\circ} \mathrm{C}$. Relative expression level of genes was calculated using the $2^{-\Delta \Delta \mathrm{Ct}}$ method (18). The sequences of the primers were as follows: FOXP3, forward 5'-CGAAGCTTA TGCCCAACCCCAGGCCTG-3', reverse 5'-CGGGATCCT CAGGGGCCAGGTGTAGGGTTG-3'; PDCD4, forward 5'-TGGATTAACTGTGCCAACCA-3', reverse 5'-TCTCAA ATGCCCTTTCATCC-3'; SQOR, forward 5'-CACTGGTGG CTGTGGTAT-3', reverse 5'-CACCCACTTTCCTCTTCAT-3'; PGGHG, forward 5'-GGTGGTCTCAGGAGGATGGA-3, reverse 5'-GGTCGGGTCAGAAGGAAGC-3'; and GAPDH, forward 5'-GTCAAGGCTGAGAACGGGAA3' and reverse 5'-AAATGAGCCCCAGCCTTCTC-3'. Each reaction was set up in triplicate. GAPDH was used as the internal control.

Western blot analysis. Cells $\left(2 \times 10^{5}\right.$ cells) were collected, washed twice with pre-cooled PBS and lysed using $100 \mu \mathrm{l}$ of pre-cooled RIPA lysis buffer containing protease inhibitor (Beyotime Institute of Biotechnology for $30 \mathrm{~min}$ on ice. Protein concentration was estimated using BCA protein assay kit (Pierce; Thermo Fisher Scientific, Inc.). Proteins (30 $\mu \mathrm{g})$ were separated by $10 \%$ SDS-PAGE and were transferred onto PVDF membranes. Membranes were blocked with 5\% skimmed milk in Tris-buffered saline containing $1 \%$ Tween 20 (TBST; pH 7.4) at room temperature for $1 \mathrm{~h}$. Membranes were incubated with primary antibodies against GAPDH (ProteinTech Group, Inc.; cat. no. 10494-1-AP; 1:4,000), FOXP3 (Abcam; cat. no. ab22510; 1:500) and PDCD4 (ProteinTech Group, Inc.; cat. no. 12587-1-AP; 1:1,000) at $4^{\circ} \mathrm{C}$ overnight. Membranes were then incubated with horseradish peroxidase-conjugated IgG secondary antibody (ProteinTech Group, Inc.; cat. no. SA00001-2; 1.400) for $1 \mathrm{~h}$ at room temperature. Enhanced chemiluminescence (Pierce; Thermo Fisher Scientific, Inc.) was used for visualization of immunoreactive proteins. ChemiDoc ${ }^{\mathrm{TM}} \mathrm{XRS}+$ System with Image $\mathrm{Lab}^{\mathrm{TM}}$ Software 4.1 (Bio-Rad Laboratories, Inc.) was used for densitometry analysis using GAPDH as internal control. 
Flow cytometry. Apoptosis was evaluated by flow cytometry. Cells were harvested and resuspended in PBS. Cells were stained with Annexin V-FITC/PI apoptosis kit (cat. no. BD 556547 Annexin V; BD Biosciences) according to the manufacturers' instructions at room temperature for $15 \mathrm{~min}$. Apoptotic cells were detected using a FACS Calibur Flow Cytometer (BD Biosciences) and analyzed using with FlowJo 10.0 software (FlowJo LLC).

RNA-Seq analysis. Two cell samples, control MDA-MB-231 cells and FOXP3-MDA-MB-231 cells, were prepared. Then, total RNA was extracted using an RNA easy Mini kit, and an on-column DNase digestion in RNase-Free DNase set (both Qiagen $\mathrm{GmbH}$ ) was used to avoid contamination by genomic DNA. A sequencing library was built and sequenced using an Illumina HiSeq 2000 by Gene Denovo Biotechnology.

Identification of differentially expressed genes (DEGs). Differential expression analysis was performed using the edge R package in $R$ between two samples (19). Genes with false discovery rates (FDRs) $<0.05$ and absolute fold-changes $\geq 2$ were considered differentially expressed genes.

Kyoto Encyclopedia of Genes and Genomes (KEGG) pathway analysis. Genes usually interact to participate in certain biological functions. Pathway-based analysis helps to further understand the biological functions of genes. KEGG (https://www.genome.jp/kegg/) is a major public pathway-related database. Pathway enrichment analysis can be used to identify significantly enriched metabolic pathways or signal transduction pathways in DEGs compared with the whole-genome background (20). Here, $\mathrm{N}$ is the number of all genes with a KEGG annotation, $n$ is the number of DEGs in $N$, $\mathrm{M}$ is the number of all genes annotated to specific pathways, and $\mathrm{m}$ is the number of DEGs in M. The calculated P-value underwent an FDR correction, and FDR $\leq 0.05$ was set as the threshold. Pathways meeting this condition were defined as significantly enriched pathways in DEGs.

Gene set enrichment analysis (GSEA). Enrichment analysis was performed using GSEA and MSigDB software (https://www.gsea-msigdb.org/gsea/msigdb/index.jsp, v7.1) to determine whether a set of genes in specific Gene Ontoloy term (http://geneontology.org/) pathways showed significant differences in two groups (21). Briefly, a gene expression matrix was constructed and genes were ranked using the signal-to-noise normalization method. Enrichment scores and P-values were calculated using the default parameters.

Kaplan-Meier plotter analysis. Survival analysis based on the mRNA expression levels of PDCD4 in breast cancer was performed using the Kaplan-Meier plotter website (www. kmplot.com), an online database that can assess the effect of 54,675 genes on the prognosis of patients with breast, ovarian, lung and gastric cancer. Briefly, the PDCD4 gene names were uploaded into the database, and the breast cancer cases included in the analysis were divided into two cohorts according the expression level of PDCD4. Patients with PDCD4 expression higher than the median were pooled into the group with high expression, while the patients with PDCD4 expression lower

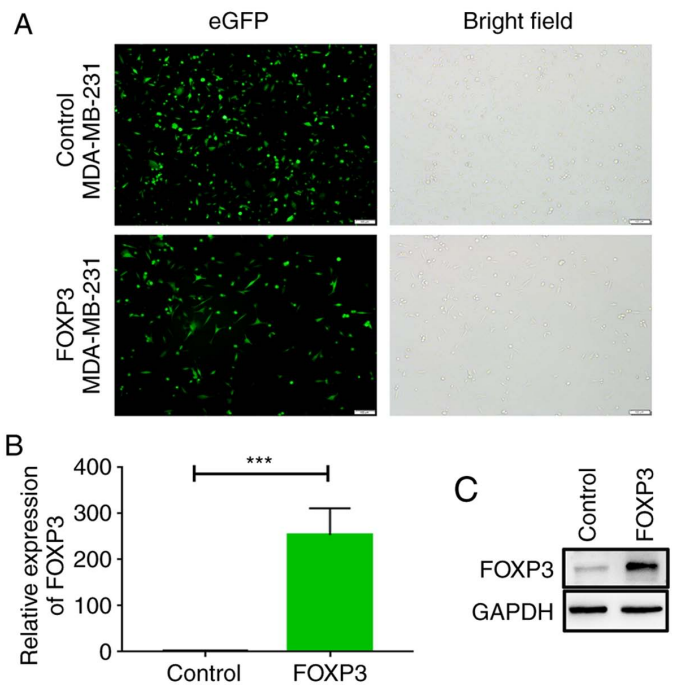

Figure 1. The generation of FOXP3-overexpressing MDA-MB-231 cells (A) MDA-MB-231 breast cancer cells were infected with GFP-labeled FOXP3-overexpressing adenovirus or control adenovirus, and the bright field and eGFP expression patterns (representing cells that were effectively infected with adenovirus) were examined using fluorescence microscopy. Magnification, x20. Scale bar, $100 \mu \mathrm{m}$. (B) Evaluation of FOXP3 mRNA expression in control and FOXP3-overexpressing MDA-MB-231 cells using reverse transcription-quantitative PCR. $n=3$. (C) Evaluation of FOXP3 protein levels in control and FOXP3-overexpressing MDA-MB-231 cells by western blotting. ${ }^{* * *} \mathrm{P}<0.001$ vs. control. eGFP, enhanced green fluorescent protein; FOXP3, forkhead box P3.

than the median were pooled into the group with low expression. Hazard ratio (HR), 95\% confidence intervals and log-rank P-values were determined using the database.

Statistical analysis. Statistical analysis between two groups of samples was performed using unpaired Student's t-test by SPSS version 16 software (SPSS Inc.) and expressed as mean \pm SEM from three independent replicates. A value of $\mathrm{P}<0.05$ was considered to indicate a statistically significant difference. The statistical tests were two-sided. The in vitro experiments were repeated at least three times. Relapse-free survival (RFS) and overall survival (OS) rates of patients in different cohorts were assessed by Kaplan Meier plots. The hazard ratio (HR) and log-rank P-values were calculated using the aforementioned databases. PDCD4 mRNA levels in different stages (according to the Scarff-Bloom-Richardson SBR grading system) were determined by Dunnett's or Tukey's post hoc test following ANOVA. Pearson's correlation coefficient was used to calculate the correlation between different genes. Correlation analysis of FOXP3 expression and PDCD4 were performed by The Cancer Genome Atlas dataset (TCGA; http://www.cancer.gov/).

\section{Results}

Generation of FOXP3-overexpressing MDA-MB-231 cells. To explore the function of FOXP 3 in breast cancer cells, MDA-MB-231 breast cancer cells were infected with green fluorescent protein (GFP)-labeled FOXP3-overexpressing adenovirus, and puromycin was used to select positive cells. As shown in Fig. 1A, cells expressed GFP, which indicated that these cells were successfully infected with adenovirus. RT-qPCR and western blotting was then conducted to further 
A
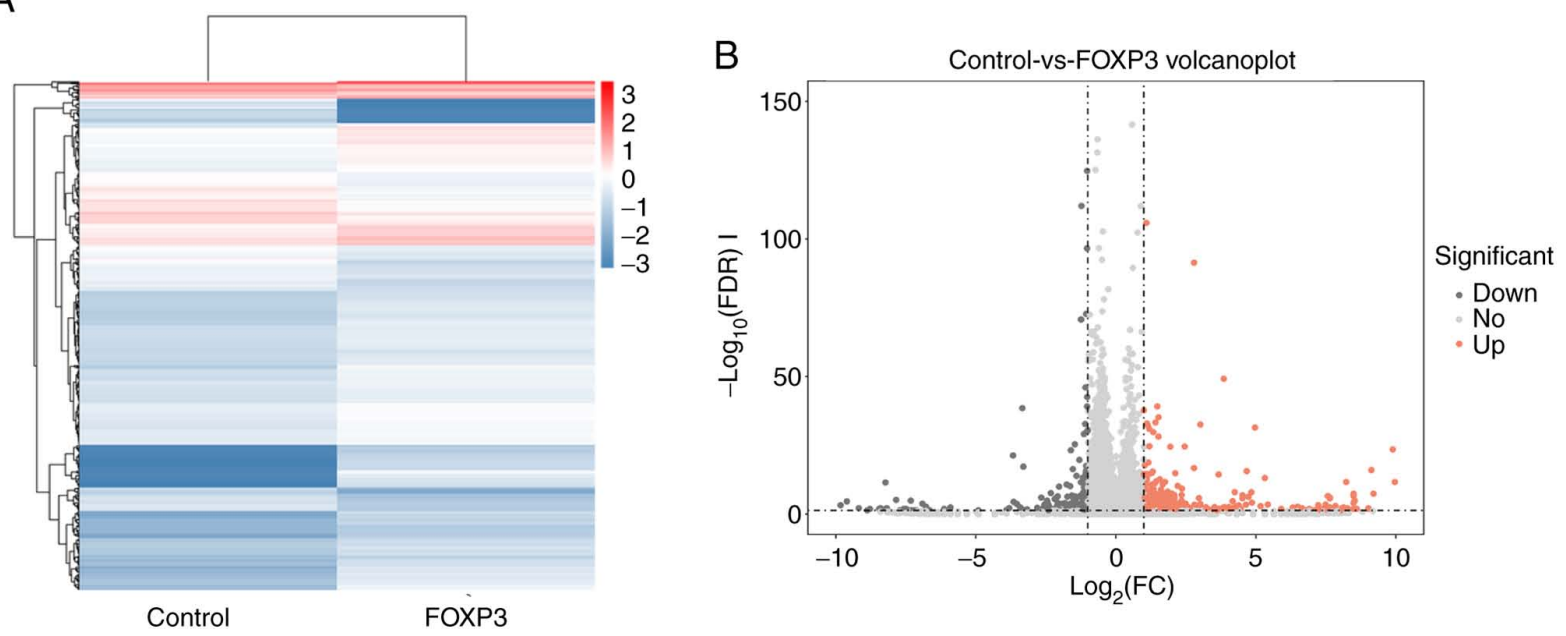

Figure 2. Identification of DEGs between FOXP3-overexpressing MDA-MB-231 and control MDA-MB-231 cells. (A) Hierarchical cluster of the differential expression levels between FOXP3-overexpressing MDA-MB-231 cells and control MDA-MB-231 cells. The color scale represents the log 10 expression values; red indicates high expression levels, and blue indicates to low expression levels. (B) The scatter plot of DEGs. Each point represents a gene. Dark gray points represent upregulated genes. Red points represent downregulated genes. Light gray points represent genes without differential expression. FC $>2$. FOXP3, forkhead box P3; DEGs, differentially expressed genes; FC, fold-change; FDR, false discovery rate; down, downregulated; no, no difference; up, upregulated.

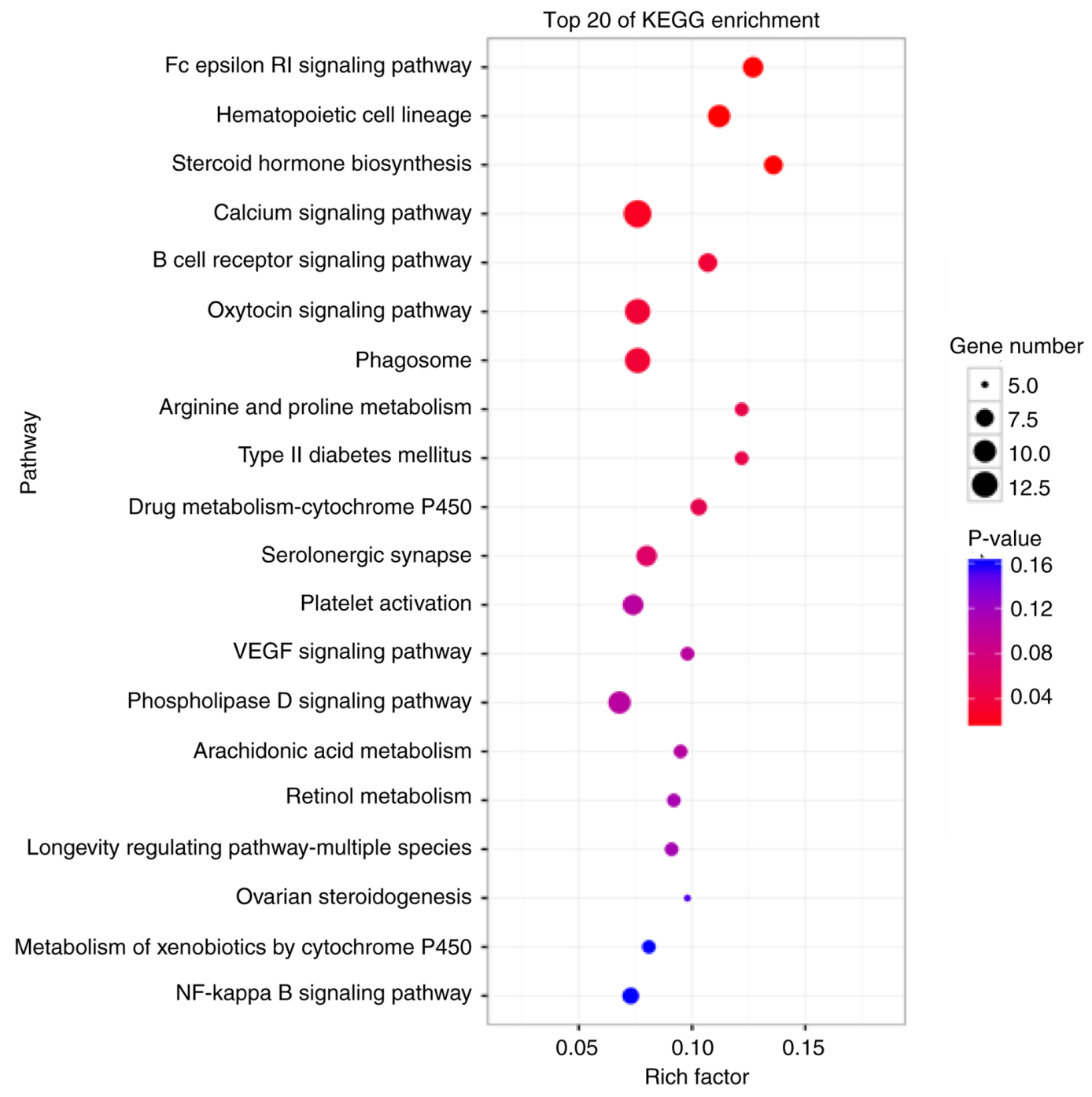

Figure 3. KEGG analysis of DEGs. Top 20 significantly enriched KEGG pathways of FOXP3-regulated DEGs. Each point represents a specific KEGG signaling pathway, and the size of the point indicates the number of DEGs enriched in each pathway. Rich factor refers to the enrichment level of DEGs enriched in the pathway. A Q value that is closer to zero indicates that the enrichment is more significant. KEGG, Kyoto Encyclopedia of Genes and Genomes; FOXP3, forkhead box P3; DEGs, differentially expressed genes. 
A
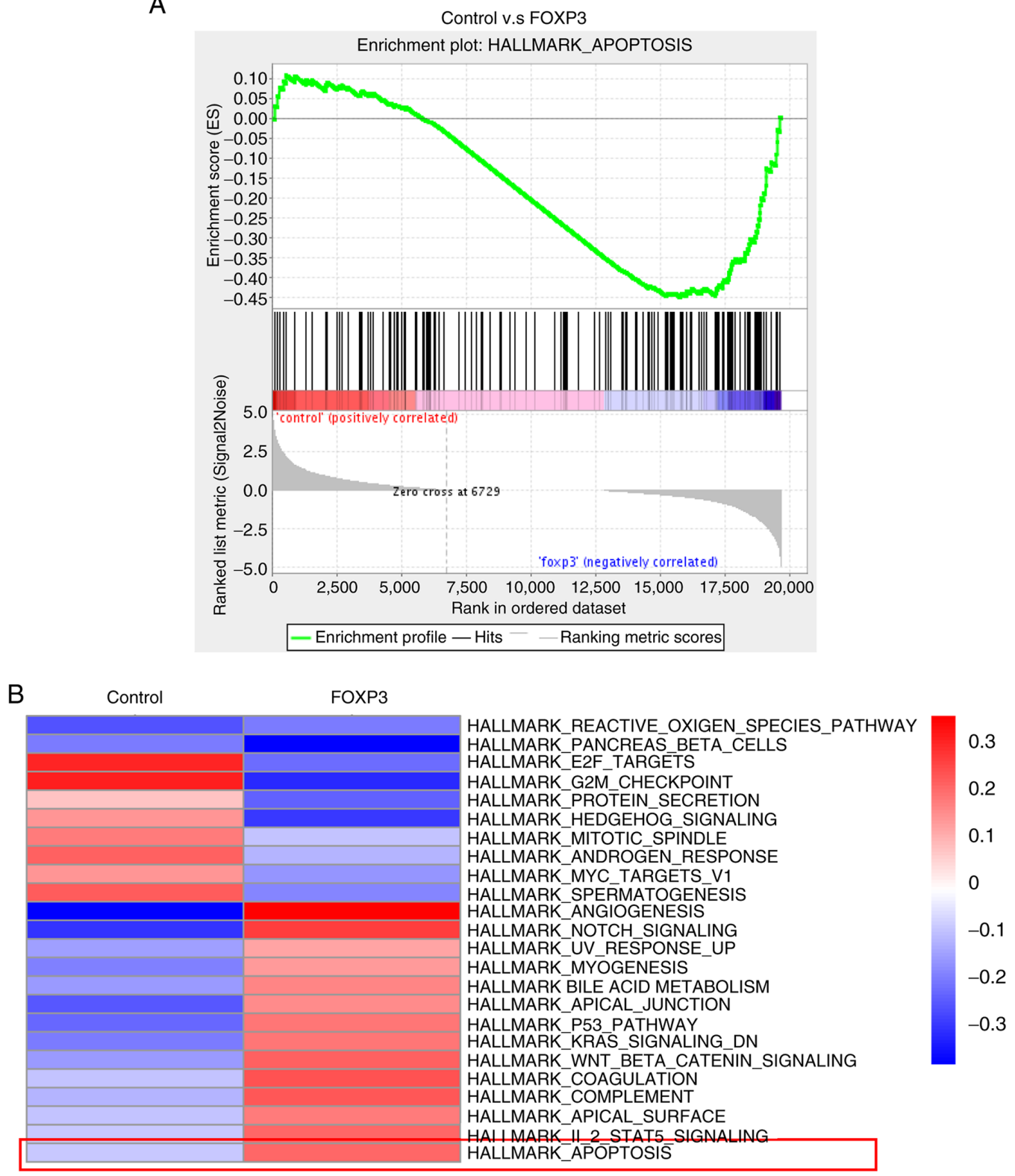

Figure 4. FOXP3 is associated with breast cancer apoptosis. (A) Gene set enrichment analysis demonstrated the enrichment of gene sets related to apoptosis in the ranked gene list of FOXP3-overexpressing MDA-MB-231 cells vs. control cells. (B) Functional enrichment-based clustering analysis between FOXP3-overexpressing MDA-MB-231 cells and wild-type MDA-MB-231 cells. FOXP3, forkhead box P3. Differences in pathway activities scored per sample using GSVA. Blue, downregulation pathway; Red, upregulation pathway.

verify the FOXP3 expression levels. The results demonstrated that FOXP3 mRNA levels in FOXP3-overexpressing MDA-MB-231 cells were significantly higher compared with those in control cells $(\mathrm{P}<0.001$; Fig. 1B). Moreover, western blot assays showed that control MDA-MB-231 cells expressed low levels of FOXP3, and FOXP3-MDA-MB-231 cells exhibited higher FOXP3 expression (Fig. 1C). Collectively, these results suggested that a FOXP3-overexpressing MDA-MB-231 cell line was successfully constructed, and this cell line and the control cells were used in the subsequent studies.

Identification of DEGs between FOXP3-overexpressing $M D A-M B-231$ and control MDA-MB-231 cells. To identify genes regulated by FOXP3, total RNA was obtained from the FOXP3-overexpressing MDA-MB-231 cells and wild-type
MDA-MB-231 cells and was subjected to RNA-seq. Then, global gene expression analysis was performed comparing FOXP3-MDA-MB-231 cells and wild-type cells. A heat map of DEGs was constructed, and expression changes in genes are shown by hierarchical cluster analysis (Fig. 2A). The RT-PCR analysis showed the high expression of three differentially expressed genes (PGGHG, SQOR and PDCD4 genes) in the RNA-seq analysis of FOXP3-overexpressing MDA-MB-231 cells (Fig. S1). Furthermore, a volcano plot shows that 6,285 genes were upregulated and 6,013 genes were downregulated in FOXP3-MDA-MB-231 cells (Fig. 2B).

KEGG analysis of DEGs. To further specify the interactions of the pathways and to clarify the biological functions of FOXP3, KEGG database analysis, which can be used to find frequently and 

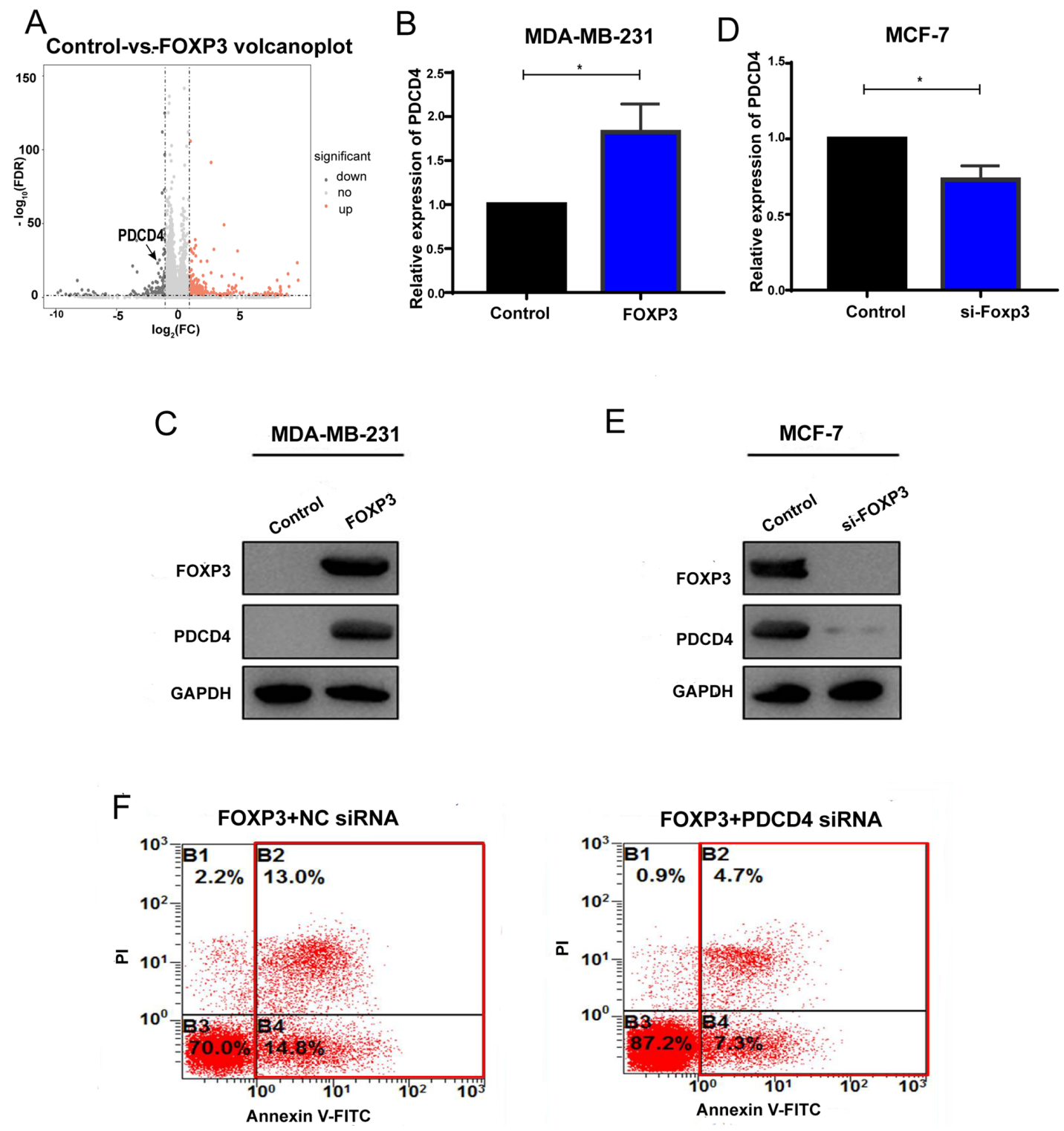

Figure 5. FOXP3 upregulates PDCD4. (A) Hierarchical cluster of the differential expression levels between FOXP3-overexpressing MDA-MB-231 cells and wild-type MDA-MB-231 cells. PDCD4 was included in the upregulated genes. (B and C) MDA-MB-231 cells were transfected with pcDNA3.1-FOXP3 or a control vector, and RT-qPCR and western blotting were conducted to detect the (B) mRNA and (C) protein levels of PDCD4. n=3. (D and E) MCF-7 cells were transfected with FOXP3 siRNA or scrambled RNA, and RT-qPCR and western blotting were conducted to detect the (D) mRNA and (E) protein levels of PDCD4. $n=3$. (F) Apoptosis was evaluated in FOXP3-overexpressing cells transfected with PDCD4 siRNA or NC siRNA. "P<0.05. FOXP3, forkhead box P3; PDCD4, programmed cell death 4; RT-q, reverse transcription-quantitative; si, small interfering; NC, negative control; FC, fold-change; FDR, false discovery rate; down, downregulated; no, no difference; up, upregulated.

significantly enriched pathways, was performed (22). The results demonstrated that the DEGs were enriched in several signaling pathways, and the main enriched terms were 'phagosome', 'oxytocin signaling pathway', 'serolonergic synapse', 'phospholipase D signaling pathway', 'platelet activation' and 'drug metabolism-cytochrome P450’ (Fig. 3).

FOXP3 is associated with breast cancer apoptosis. To determine whether FOXP3 could regulate apoptosis-related genes, GSEA was conducted. The results revealed the enrichment of a gene signature related to apoptosis in FOXP3-overexpressing MDA-MB-231 cells compared with wild-type cells (Fig. 4A). Functional enrichment-based clustering analysis was also conducted and it was demonstrated that, compared with wild-type cells, the upregulated genes in FOXP3-overexpressing MDA-MB-231 cells were also involved in apoptosis (Fig. 4B). Taken together, these data suggested that FOXP3 is associated with breast cancer apoptosis.

FOXP3 upregulates PDCD4. As aforementioned, FOXP3 expression levels were associated with apoptosis. To identify the role of FOXP3 in breast cancer apoptosis, the DEGs in FOXP3-overexpressing MDA-MB-231 cells and wild-type cells were further analyzed. It was revealed that PDCD4, a key mediator of apoptosis (23), was upregulated in FOXP3-overexpressing MDA-MB-231 cells (Fig. 5A). These results indicated that FOXP3 might be a regulator of PDCD4 in breast cancer cells. To further investigate the regulatory role of FOXP3 in PDCD4 expression in breast cancer cell lines, 
A

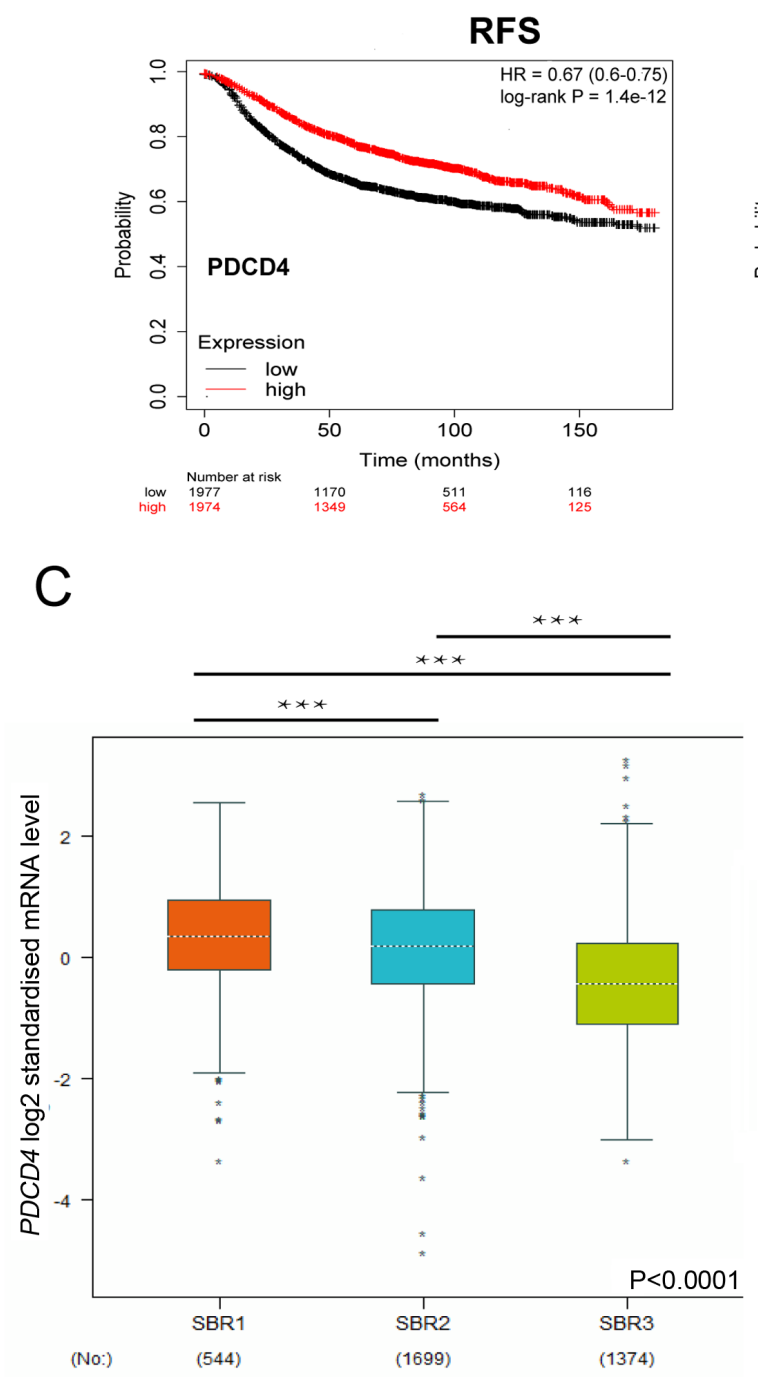

B

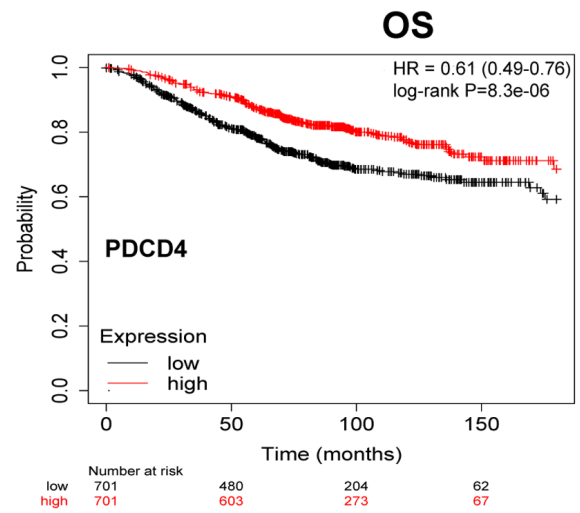

D

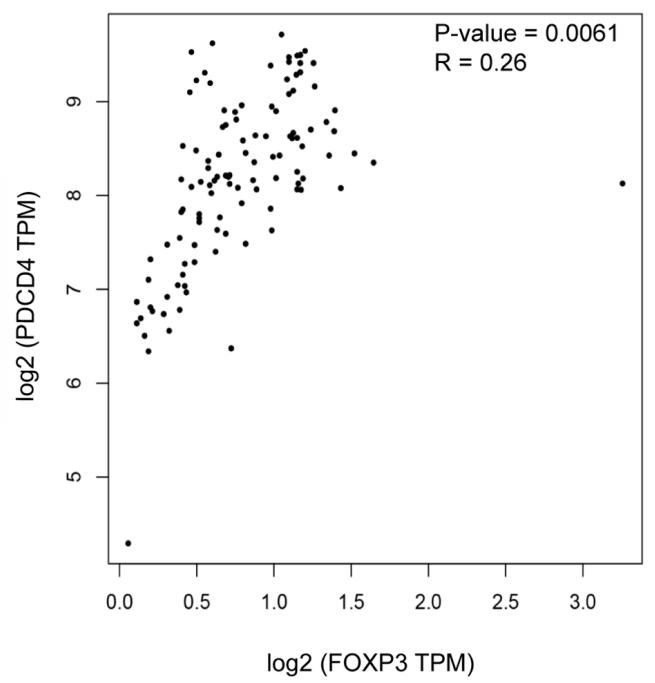

Figure 6. PDCD4 is associated with breast cancer progression. (A) Kaplan-Meier survival curves for RFS in patients with high or low PDCD4. n=3,951. $\mathrm{HR}=-0.67$; log-rank $\mathrm{P}=\mathrm{P}=1.4 \times 10^{-12}$ (B) Kaplan-Meier survival curves for OS of patients with high and low PDCD4 expression. $\mathrm{n}=1,402$. HR=0.61; log-rank $\mathrm{P}=8.3 \times 10^{-6}$. (C) PDCD4 mRNA levels in different stages of breast cancer from a public database. $\mathrm{n}=3,617$ breast cancer patients. (D) The expression of FOXP3 was positively correlated with PDCD4 in The Cancer Genome Atlas dataset. Pearson's correlation coefficient, $\mathrm{P}=0.0061, \mathrm{R}=0.26$. ${ }^{* * *} \mathrm{P}<0.0001$. RFS, relapse-free survival; OS, overall survival; TPM, transcripts per million; SBR, Scarff-Bloom-Richardson; PDCD4, programmed cell death 4; HR, hazard ratio.

RT-qPCR and western blotting were performed to analyze PDCD4 expression in breast cancer cells that gained or lost FOXP3 expression. It was reported that the ectopic expression of FOXP3 in MDA-MB-231 cells upregulated PDCD4 expression and that silencing endogenous FOXP3 in MCF-7 cells downregulated PDCD4 expression at both the mRNA and protein levels (Fig. 5B-E). Then, cell apoptosis was evaluated in FOXP3-overexpressing cells transfected with PDCD4 siRNA or NC siRNA by flow cytometry analysis. The results demonstrated that, compared with NC siRNA group, knockdown of PDCD4 in FOXP3-overexpressing cells could decrease apoptosis (Fig. 5F). Taken together, these results indicated that FOXP3 may promote PDCD4 expression in breast cancer.

PDCD4 is negatively correlated with breast cancer progression. To further identify the role of PDCD4 in breast cancer, the associated between PDCD4 expression and survival was analyzed for breast cancer samples using Kaplan-Meier plotter. As shown in Fig. 6A and B, high PDCD4 expression was a protective factor for breast cancer $\mathrm{RFS}(\mathrm{HR}=0.67$; log-rank $\mathrm{P}<0.001)$ and $\mathrm{OS}(\mathrm{HR}=0.61$; log-rank $\mathrm{P}<0.001)$. In addition, by analyzing the expression of PDCD4 in breast cancer at different stages, it was demonstrated that PDCD4 was associated with the breast cancer stage (Fig. 6C). Finally, it was reported that the expression of FOXP3 was positively correlated with PDCD4 in TCGA dataset (Fig. 6D). Collectively, these results indicated that PDCD4 may have a suppressive role of breast cancer progression and that FOXP3 might enhance breast cancer apoptosis by upregulating PDCD4.

\section{Discussion}

Breast cancer is the most frequently diagnosed cancer in women worldwide. Due to its high invasiveness and metastasis, breast cancer is the leading cause of cancer-associated 
death in women $(24,25)$. In China, the breast cancer is also the most common cancer in women, and it is occurring with increasing frequency in younger women $(26,27)$. Our previous study has shown that FOXP3 is an important breast cancer suppressor gene (28) and its loss of function or mutation is closely associated with the development and prognosis of breast cancer $(12,15,29,30)$. As a transcription factor, FOXP3 mainly exerts its anticancer function by regulating the expression of its downstream target genes $(31,32)$. FOXP3 can inhibit the proliferation of breast cancer cells by inhibiting the expression of oncogenes, such as HER2 and S-phase kinase-associated protein $2(14,33)$. FOXP3 can also inhibit the metastasis of breast cancer by inhibiting the expression of CD44 and C-X-C chemokine receptor type 4 (16,34). In addition, it has been reported that FOXP3 can inhibit breast cancer angiogenesis by downregulating VEGF (15). Therefore, the confirmation and increased understanding of the target genes regulated by FOXP3 in breast cancer is critical, as it will be helpful to improve our understanding of the anticancer function of FOXP3 and its clinical application.

To further investigate the role of FOXP3 in breast cancer progression, the present study constructed a FOXP3-overexpressing MDA-MB-231 cell line. RNA-seq revealed that apoptosis-related genes were significantly enriched in FOXP3-overexpressing MDA-MB-231 cells, which suggested that FOXP3 may play a role in breast cancer cell apoptosis.

The balance of cell proliferation and death is the key to the maintenance of homeostasis $(35,36)$. Abnormal cell proliferation and apoptosis are important causes of numerous diseases such as Hirschsprung's disease and cancers (37). PDCD4 is a key molecule in apoptosis, which can inhibit cell growth, promoted by downregulating the expression of MAP4K1 (23) or by inhibiting the interaction between eukaryotic initiation factor 4A-I (EIF)4A1 and EIF4G $(38,39)$. Studies have revealed that PDCD4 is a tumor suppressor gene that can inhibit tumor progression by promoting apoptosis $(40,41)$. Liu et al (42) first reported that FOXP3 could promote breast cancer apoptosis by inducing microRNA-146 expression, which inhibited NF- $\kappa \mathrm{B}$ activation. The present study reported another molecular mechanism by which FOXP3 induces apoptosis. Sequencing analysis demonstrated that PDCD4 is highly expressed in FOXP3-overexpressing MDA-MB-231 cells, and that FOXP3 promotes breast cancer apoptosis by upregulating PDCD4 expression. The current study revealed that FOXP3 can promote the expression of PDCD4; however, the specific mechanism by which FOXP3 upregulates PDCD4 requires further study.

The limitation of the present study was that RNA-seq data was performed with one sample per group; however, PCR was performed to further confirm the results of the existing RNA-seq analysis. The qPCR results confirmed that PDCD4 was upregulated in FOXP3-overexpressing MDA-MB-231 cells. In addition, the overexpression and knockdown of FOXP3 was not performed in a same breast cancer cell line. However, our previously studies confirmed that MDA-MB-231 is a FOXP3 negative cell line, and MCF-7 is FOXP3-positive cell line $(14,16,33)$. Therefore, the present study overexpressed FOXP3 in MDA-MB-231 and knocked down FOXP3 expression in MCF-7 cells to explore the effect of FOXP3 on PDCD4 expression. The results indicated that overexpressing FOXP3 in MDA-MB-231 cells resulted in the upregulation of PDCD4 at both the mRNA and protein levels, while siRNA-mediated silencing of endogenous FOXP3 in MCF-7 cells resulted in the downregulation of PDCD4, which indicated that FOXP3 can promote PDCD4 expression in breast cancer cells.

In conclusion, DEGs were identified in FOXP3-overexpressing MDA-MB-231 cells compared with wild-type MDA-MB-231 cells using RNA-Seq analysis, and KEGG pathway analysis was performed to examine the roles of FOXP3 in breast cancer. Notably, it was demonstrated that the expression level of FOXP3 is closely associated with apoptosis using bioinformatics analysis. Furthermore, it was confirmed that PDCD4 expression, a key molecule of apoptosis (23), can be promoted by FOXP3. Therefore, the present study provides insights into the roles of FOXP3 in breast cancer, suggesting that FOXP3 can induce breast cancer apoptosis by promoting the expression of PDCD4. Ultimately, this provides novel insights into the anticancer function of FOXP3.

\section{Acknowledgements}

The authors would like to thank Guangzhou Gene Denovo Biotechnology Co., Ltd for assisting in sequencing.

\section{Funding}

This work was funded by the National Natural Science Foundation of China (grant nos. 81902678, 81672864, $81702590,81802632,81672800$ and 81673020) and the Key Research and Development Program of Shaanxi Province (grant nos. 2017ZDCXL-SF-01-03 and 2017SF-149).

\section{Availability of data and materials}

The datasets used and/or analyzed during the present study are available from the corresponding author on reasonable request.

\section{Authors' contribution}

JL and SW conceived and designed the study. YX and DF drafted the manuscript. JL and CZ contributed to the later revision and finalization of this paper. $\mathrm{YX}$ and $\mathrm{CZ}$ constructed the FOXP3 transgenic MDA-MB-231 cell line. DF, JH and LZ performed the molecular biological experiments. YX, CZ, SW and HZ contributed to the bioinformatics analysis. YZ contributed to data collection preliminary analysis. YZ and $\mathrm{CZ}$ reviewed and edited the manuscript. All authors read and approved the final manuscript.

\section{Ethics approval and consent to participate}

Not applicable.

\section{Patient consent for publication}

Not applicable.

\section{Competing interests}

The authors declare that they have no competing interests. 


\section{References}

1. Deng C, Zhang Q, Jia M, Zhao J, Sun X, Gong T and Zhang Z: Tumors and their microenvironment dual-targeting chemotherapy with local immune adjuvant therapy for effective antitumor immunity against breast cancer. Adv Sci (Weinh) 6 : 1801868, 2019.

2. Bray F, Ferlay J, Soerjomataram I, Siegel RL, Torre LA and Jemal A: Global cancer statistics 2018: GLOBOCAN estimates of incidence and mortality worldwide for 36 cancers in 185 countries. CA Cancer J Clin 68: 394-424, 2018.

3. Jemal A, Siegel R, Xu J and Ward E: Cancer statistics, 2010 CA Cancer J Clin 60: 277-300, 2010.

4. Waks AG and Winer EP: Breast cancer treatment: A review. JAMA 321: 288-300, 2019

5. Torre LA, Bray F, Siegel RL, Ferlay J, Lortet-Tieulent J and Jemal A Global cancer statistics, 2012. CA Cancer J Clin 65: 87-108, 2015.

6. Sun YS, Zhao Z, Yang ZN, Xu F, Lu HJ, Zhu ZY, Shi W, Jiang J, Yao PP and Zhu HP: Risk factors and preventions of breast cancer. Int J Biol Sci 13: 1387-1397, 2017.

7. Pranavathiyani G, Thanmalagan RR, Leimarembi Devi N and Venkatesan A: Integrated transcriptome interactome study of oncogenes and tumor suppressor genes in breast cancer. Genes Dis 6: 78-87, 2018.

8. Brunkow ME, Jeffery EW, Hjerrild KA, Paeper B, Clark LB, Yasayko SA, Wilkinson JE, Galas D, Ziegler SF and Ramsdell F: Disruption of a new forkhead/winged-helix protein, scurfin, results in the fatal lymphoproliferative disorder of the scurfy mouse. Nat Genet 27: 68-73, 2001.

9. Rudensky AY: Regulatory T cells and Foxp3. Immunol Rev 241: 260-268,2011.

10. Gregorczyk I and Maslanka T: Significant expression of Foxp3 in murine extrathymic CD4+CD8+ double positive T cells. Pol J Vet Sci 20: 815-817, 2017.

11. Pierini A, Nishikii H, Baker J, Kimura T, Kwon HS, Pan Y, Chen Y, Alvarez M, Strober W, Velardi A, et al: Foxp3 ${ }^{+}$regulatory $\mathrm{T}$ cells maintain the bone marrow microenvironment for B cell lymphopoiesis. Nat Commun 8: 15068, 2017.

12. Douglass S, Ali S, Meeson AP, Browell D and Kirby JA: The role of FOXP3 in the development and metastatic spread of breast cancer. Cancer Metastasis Rev 31: 843-854, 2012.

13. Karanikas V, Speletas M, Zamanakou M, Kalala F, Loules G, Kerenidi T, Barda AK, Gourgoulianis KI and Germenis AE: Foxp3 expression in human cancer cells. J Transl Med 6: 19, 2008

14. Zuo T, Wang L, Morrison C, Chang X, Zhang H, Li W, Liu Y, Wang Y, Liu X, Chan MW, et al: FOXP3 is an X-linked breast cancer suppressor gene and an important repressor of the HER-2/ErbB2 oncogene. Cell 129: 1275-1286, 2007.

15. Li X, Gao Y, Li J, Zhang K, Han J, Li W, Hao Q, Zhang W, Wang S, Zeng $C$, et al: $F O X P 3$ inhibits angiogenesis by downregulating VEGF in breast cancer. Cell Death Dis 9: 744, 2018.

16. Zhang C, Xu Y, Hao Q, Wang S, Li H, Li J, Gao Y, Li M, Li W, Xue X, et al: FOXP3 suppresses breast cancer metastasis through downregulation of CD44. Int J Cancer 137: 1279-1290, 2015.

17. Yuan H, Xin S, Huang Y, Bao Y, Jiang H, Zhou L, Ren X, Li L, Wang Q and Zhang J: Downregulation of PDCD4 by miR-21 suppresses tumor transformation and proliferation in a nude mouse renal cancer model. Oncol Lett 14: 3371-3378, 2017.

18. Livak KJ and Schmittgen TD: Analysis of relative gene expression data using real-time quantitative PCR and the 2(-Delta Delta C(T)) method. Methods 25: 402-408, 2001

19. Robinson MD, McCarthy DJ and Smyth GK: EdgeR: A Bioconductor package for differential expression analysis of digital gene expression data. Bioinformatics 26: 139-140, 2010

20. Kanehisa M and Goto S: KEGG: Kyoto encyclopedia of genes and genomes. Nucleic Acids Res 28: 27-30, 2000.

21. Subramanian A, Tamayo P, Mootha VK, Mukherjee S, Ebert BL, Gillette MA, Paulovich A, Pomeroy SL, Golub TR, Lander ES and Mesirov JP: Gene set enrichment analysis: A knowledge-based approach for interpreting genome-wide expression profiles. Proc Natl Acad Sci USA 102: 15545-15550, 2005.

22. Mao X, Cai T, Olyarchuk JG and Wei L: Automated genome annotation and pathway identification using the KEGG Orthology (KO) as a controlled vocabulary. Bioinformatics 21: 3787-3793, 2005

23. Yang HS, Matthews CP, Clair T, Wang Q, Baker AR, Li CC, Tan TH and Colburn NH: Tumorigenesis suppressor Pdcd4 down-regulates mitogen-activated protein kinase kinase kinase kinase 1 expression to suppress colon carcinoma cell invasion. Mol Cell Biol 26: 1297-1306, 2006.
24. Ghislain I, Zikos E, Coens C, Quinten C, Balta V, Tryfonidis K, Piccart M, Zardavas D, Nagele E, Bjelic-Radisic V, et al: Health-related quality of life in locally advanced and metastatic breast cancer: Methodological and clinical issues in randomised controlled trials. Lancet Oncol 17: e294-e304, 2016.

25. Gao Y, Wang Z, Hao Q, Li W, Xu Y, Zhang J, Zhang W, Wang S, Liu S, Li M, et al: Loss of ER $\alpha$ induces amoeboid-like migration of breast cancer cells by downregulating vinculin. Nat Commun 8: 14483, 2017.

26. Qin H, Yu H, Sheng J, Zhang D, Shen N, Liu L, Tang Z and Chen X: PI3Kgamma inhibitor attenuates immunosuppressive effect of poly(1-Glutamic Acid)-combretastatin A4 conjugate in metastatic breast cancer. Adv Sci (Weinh) 6: 1900327, 2019.

27. Fan L, Strasser-Weippl K, Li JJ, St Louis J, Finkelstein DM, Yu KD, Chen WQ, Shao ZM and Goss PE: Breast cancer in China. Lancet Oncol 15: e279-289, 2014.

28. Gao Y, Li X, Shu Z, Zhang K, Xue X, Li W, Hao Q, Wang Z, Zhang W, Wang S, et al: Nuclear galectin-1-FOXP3 interaction dampens the tumor-suppressive properties of FOXP3 in breast cancer. Cell Death Dis 9: 416, 2018.

29. Tian T, Wang M, Zheng Y, Yang T, Zhu W, Li H, Lin S, Liu K, $\mathrm{Xu}$ P, Deng Y, et al: Association of two FOXP3 polymorphisms with breast cancer susceptibility in Chinese Han women. Cancer Manag Res 10: 867-872, 2018.

30. Recouvreux MS, Grasso EN, Echeverria PC, Rocha-Viegas L, Castilla LH, Schere-Levy C, Tocci JM, Kordon EC and Rubinstein N: RUNX1 and FOXP3 interplay regulates expression of breast cancer related genes. Oncotarget 7: 6552-6565, 2016.

31. Katoh H, Zheng P and Liu Y: Signalling through FOXP3 as an X-linked tumor suppressor. Int J Biochem Cell Biol 42: 1784-1787, 2010.

32. Lopes JE, Torgerson TR, Schubert LA, Anover SD, Ocheltree EL, Ochs HD and Ziegler SF: Analysis of FOXP3 reveals multiple domains required for its function as a transcriptional repressor. J Immunol 177: 3133-3142, 2006.

33. Zuo T, Liu R, Zhang H, Chang X, Liu Y, Wang L, Zheng P and Liu Y: FOXP3 is a novel transcriptional repressor for the breast cancer oncogene SKP2. J Clin Invest 117: 3765-3773, 2007.

34. Douglass S, Meeson AP, Overbeck-Zubrzycka D, Brain JG, Bennett MR, Lamb CA, Lennard TW, Browell D, Ali S and Kirby JA: Breast cancer metastasis: Demonstration that FOXP3 regulates CXCR4 expression and the response to CXCL12. J Pathol 234: 74-85, 2014

35. Abraha AM and Ketema EB: Apoptotic pathways as a therapeutic target for colorectal cancer treatment. World J Gastrointest Oncol 8: 583-591, 2016.

36. Hipfner DR and Cohen SM: Connecting proliferation and apoptosis in development and disease. Nat Rev Mol Cell Biol 5: 805-815, 2004.

37. Gudipaty SA, Conner CM, Rosenblatt J and Montell DJ: Unconventional ways to live and die: Cell death and survival in development, homeostasis, and disease. Annu Rev Cell Dev Biol 34: 311-332, 2018.

38. Modelska A, Turro E, Russell R, Beaton J, Sbarrato T, Spriggs K, Miller J, Graf S, Provenzano E, Blows F, et al: The malignant phenotype in breast cancer is driven by eIF4A1-mediated changes in the translational landscape. Cell Death Dis 6: e1603, 2015.

39. Goggin MM, Nelsen CJ, Kimball SR, Jefferson LS, Morley SJ and Albrecht JH: Rapamycin-sensitive induction of eukaryotic initiation factor $4 \mathrm{~F}$ in regenerating mouse liver. Hepatology 40: 537-544, 2004.

40. Song X, Zhang X, Wang X, Zhu F, Guo C, Wang Q, Shi Y, Wang J, Chen Y and Zhang L: Tumor suppressor gene PDCD4 negatively regulates autophagy by inhibiting the expression of autophagy-related gene ATG5. Autophagy 9: 743-755, 2013

41. Wang X, Li Y, Wan L, Liu Y, Sun Y, Liu Y, Shi Y, Zhang L, Zhou H, Wang J, et al: Downregulation of PDCD4 induced by progesterone is mediated by the PI3K/AKT signaling pathway in human endometrial cancer cells. Oncol Rep 42: 849-856, 2019.

42. Liu R, Liu C, Chen D, Yang WH, Liu X, Liu CG, Dugas CM, Tang F, Zheng P, Liu Y and Wang L: FOXP3 controls an miR-146/NF-KB negative feedback loop that inhibits apoptosis in breast cancer cells. Cancer Res 75: 1703-1713, 2015.

This work is licensed under a Creative Commons Attribution-NonCommercial-NoDerivatives 4.0 International (CC BY-NC-ND 4.0) License. 